\title{
Hindlimb torsional alignment changes in growing rabbits after patellar dislocation
}

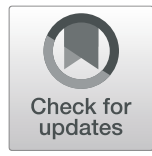

Jinghui Niu', Qi Qi ${ }^{2}$, Kang Piao ${ }^{1}$, Kuo Hao ${ }^{1}$, Iftekhar Sharif ${ }^{1}$ and Fei Wang ${ }^{1 *}$

\begin{abstract}
Background: Torsional malalignment has been considered as a risk factor for patellar dislocation. But the influence of patellar dislocation for torsional alignment development remains unknown. The present study aims to investigate whether the torsional alteration of the hindlimb occurs after patellar dislocation in growing rabbits.

Methods: In the present study, 30 one-month-old rabbits were included. The experimental group consisted of 30 left knees of rabbits which underwent patellar lateral dislocation. The control group consisted of 30 right knees of the rabbits which no surgical procedure was performed. The Computed Tomography (CT) scan was performed after the surgery and at the point the rabbits were skeletal mature ( 5 months post-surgery). The angles of femoral version and tibial torsion were measured using a three-dimensional method and analyzed between the experimental group and the control group.
\end{abstract}

Results: After the surgery, the femoral version and tibial torsion in the experimental and control group were not significantly different. However, 5 months after surgery, the angle of femoral version in the experimental group $\left(-5.50 \pm 6.13^{\circ}\right)$ was significantly different from that in the control group $\left(-10.90 \pm 4.74^{\circ}\right)(P<0.05)$. But the angle of tibial torsion in the experimental group $\left(7.17 \pm 7.25^{\circ}\right)$ and control group $\left(4.47 \pm 6.34^{\circ}\right)$ were not significantly different $(P=0.144)$.

Conclusions: From this study, patellar dislocation can lead to alteration of femoral version in growing rabbits. So patellar dislocation may affect on lower extremity alignment. These findings may develop pathology and etiology of patellar dislocation.

Keywords: Rabbits, Patellar dislocation, Growing, Bone malalignment

\section{Background}

Several anatomic factors are associated with patellar dislocation, including increased tibial tubercle-trochlear groove (TT-TG) distance, patella alta, rotational deformities, trochlear dysplasia,and patella shape [1-6]. Among the factors, rotational malalignment has been regarded as a risk factor for patellar dislocation in previous studies [2-4]. For femoral anteversion, Dejour et al. [2] found that the average femoral anteversion of normal knees was $10.8^{\circ}$, but the average femoral anteversion of knees

\footnotetext{
* Correspondence: njhxueshu@163.com

'Department of joint surgery, Hebei Medical University Third Affiliated Hospital, Shijiazhaung City, Hebei Province, China

Full list of author information is available at the end of the article
}

with patellar instability was $15.6^{\circ}(P=0.013)$. Erkocak [3] and Takagi [4] also found the patients with a history of patellar instability had a higher mean femoral anteversion, compared with the normal's. For tibial torsion, no significant difference was found between patients with patellofemoral instability and the normal $[3,5]$. Clinically, strategic choice of surgical treatment for patients with patellar instability may be influenced by the torsion of the lower limbs [7]. For patients with patellar instability who have a femoral anteversion higher than $25^{\circ}$, single patellofemoral ligament reconstruction for patellar instability may be insufficient, and derotational femoral osteotomy should be considered [7]. 
Previous studies have investigated the influence of patellar dislocation on the development of the patellofemoral joint by rabbit models [8-10]. Wang [8], Li [9], and Niu [10] found femoral trochlear dysplasia and patellar dysplasia could occur after patellar dislocation in growing rabbits. On the other hand, Kaymaz found trochlea flattening after surgery for creating patella alta in growing rabbits [11]. These studies indicated that the dysplasia of patella or femoral trochlea could be caused by an abnormal patellar position. In another animal study [12], tibial tubercle lateralization and tibial tuberosity-trochlear groove distance (TT-TG) increased after patellar dislocation.

Although low extremity malalignment is considered as a predisposing factor for patellar dislocation [2-4], the effect of patellar dislocation on low extremity alignment development has remained unclear. To our best knowledge, this is the first study focusing on the alteration of the torsional alignment after the patellar dislocation. The objectives of the present study were to elucidate the alignment alteration in the transverse plane after patellar dislocation in growing rabbits and discuss the influence of patellar dislocation on lower extremity alignment. Based on the previous animal studies, we used the rabbit model for patellar dislocation and set the null hypothesis as that early patellar dislocation could not lead to a significant difference of torsional hindlimb alignment in growing rabbits.

\section{Methods}

\section{Study Design and setting}

This study was approved by the Animal Ethics Committee of the third hospital of Hebei Medical University (Number: Z2019-006-1; Date:2019-02-25). Sixty knees from 30 healthy, 1-month-old female New Zealand white rabbits, weighing between 350 and $450 \mathrm{~g}$ (provided by the Animal Center of the Hebei Medical University), were split into two groups. The experimental group consisted of 30 left knees, which were performed patellar dislocation surgery. The control group comprised 30 right knees with no surgical procedure. All procedures performed in studies involving animals were under the Western University's Animal Care and Use Guidelines ( London, Ontario, Canada) [13].

The rabbits were kept individually in cages $(310 \times$ $550 \times 320 \mathrm{~mm})$, under controlled temperature $(22 \pm$ $2^{\circ} \mathrm{C}$ ), humidity $(55 \pm 5 \%), 12$-hour light-dark cycle (7:00 a.m. to 7:00 p.m.). The rabbits were raised with unrestricted access to standard water and food and were allowed 30 minutes of activity out of cages per day. The rabbits were euthanized by excessive anesthesia of pentobarbital sodium by injection through the ear vein at the end of the study.

\section{Surgical procedures}

The processes for making patellar dislocation models of growing rabbits have been described and proved by previous studies [8-10]. The one-month-old rabbits were administered anesthesia of ketamine hydrochloride and xylazine at a dosage of 20 and $5 \mathrm{mg} / \mathrm{kg}$ body weight by injection through the ear vein. The rabbits were fixed on the platform for surgery with spine position. Then, the left knees of the experimental group were shaved and disinfected by standard procedures. A $2.5-\mathrm{cm}$ incision was performed on the middle line of the knee skin, and the soft tissue was dissected to expose the medial retinaculum and the joint capsule. The medial retinaculum and medial joint capsule of the knees were incised about $1.5-\mathrm{cm}$, and the patella was then pushed laterally with hemostatic forcep to expose the femoral trochlea. At this time, the later joint capsule and lateral retinaculum were overlapped and sutured together. After these procedures, patellar dislocation could be seen intraoperatively (Fig. 1). The patella dislocated (the femoral trochlea was exposed) when the knee was flexed and extended. All the procedures were performed carefully to avoid cartilage and blood vessel damage. At last, the incision was interrupted sutured, and bandages were applied over the incision. CT scans were performed immediately after surgery to confirm lateral patellar dislocation. Ciprofloxacin $(10 \mathrm{mg} / \mathrm{kg}, \mathrm{PO})$ was administered three days postoperatively for prophylaxis. The sutures were taken out two weeks postoperatively. The growing rabbits achieve skeletal maturation at 6 months [14], so the rabbits were euthanized at 5 months post-surgery when they were skeletally mature after the last follow-up.

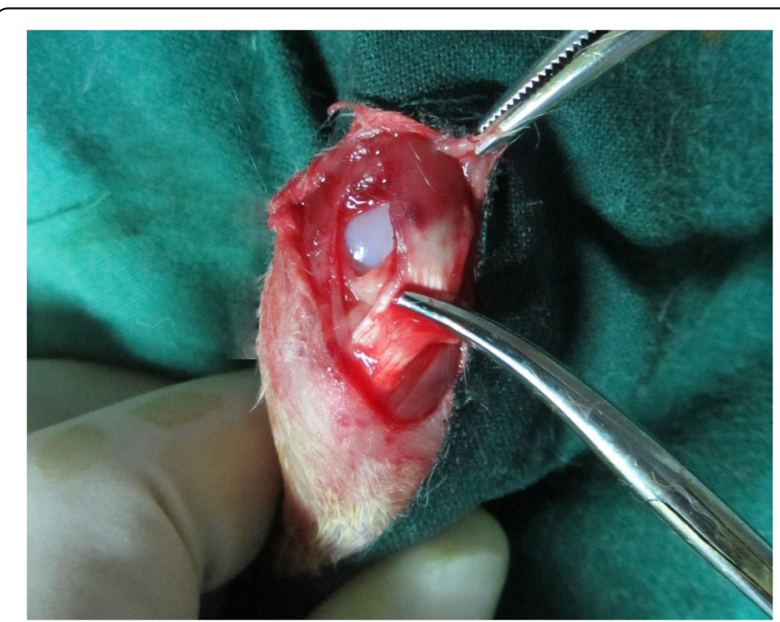

Fig. 1 The picture during surgery. The medial retinaculum and joint capsule were incised. Patella was moved laterally and femoral trochlear could be seen 


\section{CT assessment}

CT scans of the rabbits were performed immediately after the operation and 5 months postoperatively using a 16-slice CT scanner (SOMATOM Sensation 16; Siemens Medical Solutions, Erlangen, Germany). The rabbits were under anesthesia and were placed in a supine position. The hindlimb was fully extended and was fixed on a board to prevent any movements during scanning. Contiguous slices $(1.0 \mathrm{~mm})$ were obtained from the upper rim of the pelvis to the most distal part of the hindlimb. Considering the different structures of the hindlimb in rabbits and the accuracy of the measurements, the measurements were performed in a 3dimensional strategy as the previous studies showed [4, 15-19]. The CT slices were sent to RadiAnt DICOM software (Medixant Ltd., Poznan, Poland) and reconstructed for 3D models. Our measuring methods had an accuracy of $0.01^{\circ}$.

The femoral version was measured as the study by Jia showed [15]. After 3D image construction, the femur was given a superior view (Fig. 2a). The lowest point of the greater trochanter, the femoral medial condyle, and femoral lateral condyle were moved and rotated for adjustment until the lowest point of the greater trochanter were positioned in the middle between the medial and lateral femoral condyle. The three points were connected by horizontal line $\mathrm{B}$. The femoral neck version was the angle formed by the line $\mathrm{B}$ and line $\mathrm{A}$ which connecting the point of the femoral head center with the midpoint of the narrowest femoral neck part (Fig. 2b). The condition that the femoral neck is anterior to the posterior condylar line is defined as positive.

For tibial torsion measurement, as in the previous study [4], the tibia was given an inferior view after 3D image reconstruction. (Fig. 3a). The tibia was rotated and moved until the tibia shaft was almost covered by the ankle joint. The most posterior points of the medial and lateral tibia condyle were connected by Line D. Tibial torsion was measured by the angle between the line $\mathrm{D}$ and the line $\mathrm{C}$ which was drawn through the center of lateral and medial malleoli (Fig. 3b). The condition that the ankle laterally rotated to the posterior tibial plateau is defined as positive.

\section{Statistics}

Statistical analysis was performed using SPSS version 21.0 (SPSS, IL, USA). The results are expressed as mean \pm standard deviation. Levene's test was used to evaluate the homogeneity of the data. The mean difference of femoral version and tibial torsion between the control group and the experimental group were evaluated by Student's $t$ test. A $P$ value $<0.05$ was determined as statistically significant.

For determining the intra-observer variation, observer One(QQ) repeated the observations 2 weeks after the first measurement. To determine the inter-observer variation, the observations were performed by observer One(QQ), observer Two(KP), and observer Three(KH). The observers were blinded to the grouping. Intraobserver consistency and inter-observer consistency were analyzed using the intra-class correlation coefficient (ICC). ICC higher than 0.75 was excellent, ICC lower than 0.40 was poor, ICC among 0.40 to 0.75 was fair to good [20].

Based on the previous study [4, 21], the femoral version was selected as the primary variable for sample size calculation, the difference was set as $5^{\circ}$ between the two groups, the standard deviation was assumed as $6^{\circ}$. With

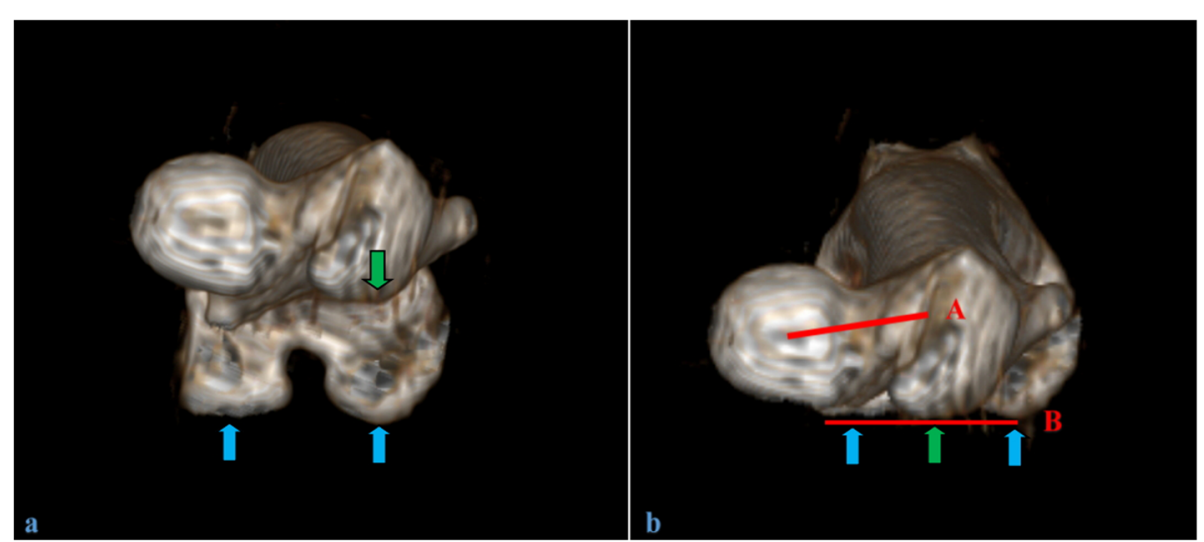

Fig. 2 The schematic diagram of femoral version angle measurement. a: The superior view of the femur after 3D reconstruction. b: The 3D femur after adjustment. Line B connects the lowest point of the greater trochanter and femoral medial and lateral condyle. Line A connects the point of the center of the femoral head with the midpoint of the narrowest femoral neck. The femoral version was measured by the angle between Line A and Line B. The green arrow indicates the lowest point of the great trochanter. The blue arrows indicate the lowest point of the femoral medial and lateral condyle 


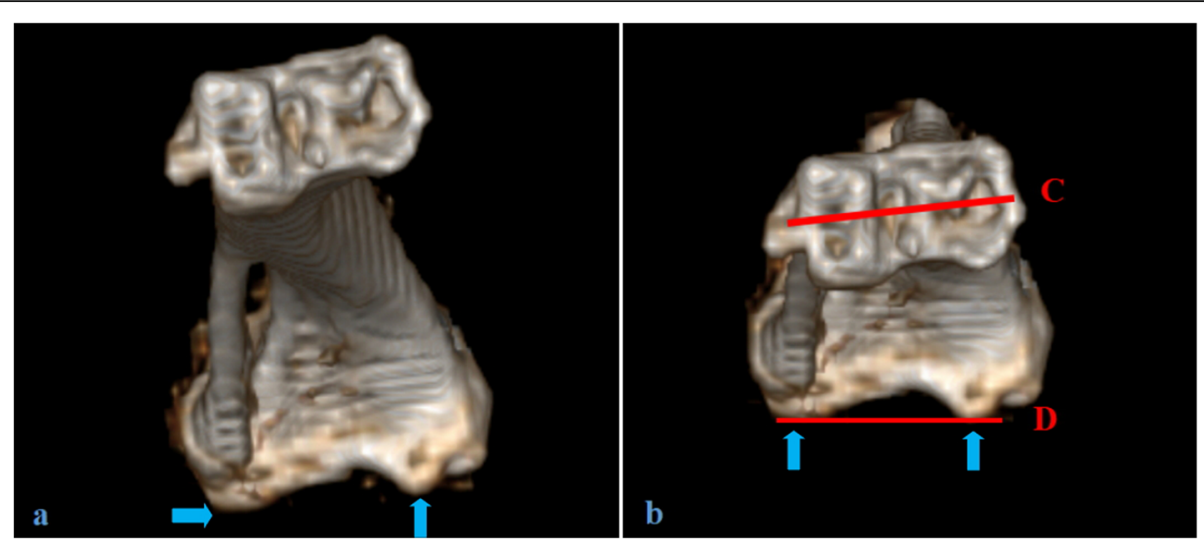

Fig. 3 The schematic diagram of tibial torsion angle measurement. a: The inferior view of the tibia after 3D reconstruction. b: The 3D tibia after adjustment. Line D connects the medial and lateral tibial condyles. Line $\mathrm{C}$ is drawn through the center of medial and lateral malleoli. The tibial torsion was measured by the angle between Line $\mathrm{C}$ and Line $\mathrm{D}$. The blue arrows indicate the lowest point of tibial medial and lateral condyle

a confidence level of $95 \%(\alpha=0.05)$ and power $(1-\beta)$ of $80 \%$, the power calculation was performed and 24 knees were needed per group.

\section{Results}

From CT scans, all the patellas dislocated laterally from femoral trochlea, which showed the patella dislocation model was achieved successfully. In this study, the femoral version and tibial torsion were measured immediately after surgery, and the values were not significantly different between the experimental group and the control group (Table 1). Two rabbits died of postoperative infection in one week after surgery. So 28 rabbits were taken CT scanning 5 months after surgery. The average weight of the 1-month-old rabbits was $0.4 \mathrm{~kg}$, and at the last follow-up, the average weight was $2.8 \mathrm{~kg}$. At that time, the femoral version of the experimental group was significantly different from that of the control group. But the tibial torsion in the experimental group and control group were not significantly different (Table 2). The intra-observer consistency and interobserver consistency were showed in Table 3.

\section{Discussion}

The most important finding of this study was that the femoral version was significantly different between the

Table 1 Measurements immediately after operation

\begin{tabular}{lcccc}
\hline Measurement $\left({ }^{\circ}\right)$ & Experimental group & Control group & $\mathbf{N}$ & $\boldsymbol{P}$ value \\
\hline Femoral version & $11.88 \pm 4.89$ & $13.50 \pm 5.51$ & 30 & 0.205 \\
Tibia torsion & $11.56 \pm 4.03$ & $12.94 \pm 3.48$ & 30 & 0.164
\end{tabular}

Significantly different: $P<0.05$. Femoral version: positive: femoral neck is anterior to posterior condylar line; negative: femoral neck is posterior to posterior condylar line. Tibia torsion: positive: ankle laterally rotated to the posterior tibial plateau; negative: ankle internally rotated to the posterior tibial plateau. experimental group and control group in growing rabbits after patellar dislocation, and the tibial torsion was not significantly different between the two groups.

The femoral version shows the relative position of the femoral neck and the transcondylar axis or coronal plane of the distal femur. Femoral anteversion refers to the anterior rotation of the femoral head from the coronal plane of the femur. And femoral retroversion is defined as the condition that the femoral neck axis locates posterior to the transcondylar axis or the coronal plane of the femur [22].

For human beings, there is $30^{\circ}$ to $40^{\circ}$ of the femoral anteversion at birth. It decreases to $10^{\circ}$ to $15^{\circ}$ when skeletally mature. Most of the alteration occurs before the age of 8 years $[23,24]$. For rabbits, there were $10^{\circ}$ of anteversion in the femur at first. The anteversion disappeared by the eighth week, and by the time the rabbits were skeletally mature, $10^{\circ}$ to $15^{\circ}$ degrees of femoral retroversion has been observed [21]. Although the decreasing trends of the femoral version development between human-beings and rabbits may look numerically similar, the femoral version between them was different. Actually, for adults, femoral retroversion is not as common as femoral anteversion. In the study by Hartel, 1070 left femurs were performed thin-slice CT scans, and 77 subjects $(7.8 \%)$ were found with the retroverted femur (range $-23.6^{\circ}-0.2^{\circ}$ ) [25]

Table 2 Measurements five months after operation

\begin{tabular}{lcccc}
\hline Measurement $\left({ }^{\circ}\right)$ & Experimental group & Control group & $\mathbf{N}$ & $\boldsymbol{P}$ value \\
\hline Femoral version & $-5.50 \pm 6.13$ & $-10.90 \pm 4.74$ & 28 & 0.001 \\
Tibia torsion & $7.17 \pm 7.25$ & $4.47 \pm 6.34$ & 28 & 0.144
\end{tabular}

Significantly different: $P<0.05$. Femoral version: positive: femoral neck is anterior to posterior condylar line; negative: femoral neck is posterior to posterior condylar line. Tibia torsion: positive: ankle laterally rotated to the posterior tibial plateau; negative: ankle internally rotated to the posterior tibial plateau. 
Table 3 Inter- and Intraobserver Reliability of the Different Measurements

\begin{tabular}{|c|c|c|c|}
\hline \multicolumn{4}{|c|}{ Intraclass Correlation Coefficient $(95 \% \mathrm{Cl})$} \\
\hline Measurements & & Immediately after surgery & 5 months postoperatively \\
\hline \multirow[t]{2}{*}{ Femoral version } & Intra-observer Reliability & $0.931(0.877-0.961)$ & $0.939(0.860-0.970)$ \\
\hline & Inter-observer Reliability & $0.912(0.868-0.944)$ & $0.870(0.807-0.917)$ \\
\hline \multirow[t]{2}{*}{ Tibia torsion } & Intra-observer Reliability & $0.838(0.733-0.902)$ & $0.971(0.953-0.984)$ \\
\hline & Inter-observer Reliability & $0.838(0.765-0.893)$ & $0.966(0.947-0.979)$ \\
\hline
\end{tabular}

The femoral version relates to the stability and function of the knee and hip joints. The abnormal femoral version affects many diseases, including torsional syndromes, fractures of the femur, hip dysplasia, LeggCalve-Perthes disease, and anterior cruciate ligament (ACL) rupture [26-30]. The femoral version also affects patellar stability. The increased femoral anteversion has been regarded as a risk factor for patellar instability, as it produces a lateralizing force on the patella [31]. The lateralizing force exists even after medial patellofemoral ligament reconstruction, contributes to the inferior clinical outcomes, even reconstruction failure [32, 33].

In this study, the femoral retroversion decreased after patellar dislocation in growing rabbits. Patellar dislocation may cause the alteration of the force direction of rectus femoris muscle. Also, we found knee or ankle lateral rotation in activities of rabbits after a patellar dislocation. The alteration of strength direction and the bone position may be the reason for the femoral version difference during growth. The version of the femur changed significantly after patellar dislocation, but the tibial torsion did not change significantly in the growing rabbits after patellar dislocation. Similar to humans, in the lower extremity, the femur may be abnormal although the tibia and fibula are well-formed or only slightly hypoplastic. And the foot may be normal despite the severe anomalies in the proximal part of the lower extremity [34].

For the previous experimental studies using rabbit models $[8-10,12]$, patellar dislocation or instability could lead to femoral dysplasia, patellar dysplasia, and higher TT-TG. A higher sulcus angle and lower trochlear depth, which indicated femoral trochlear dysplasia, were found in the rabbit knees after early patellar dislocation or instability $[8,9]$. In another study, a longer diameter and higher Wiberg-angle of the patellas were found after patellar instability, which showed patellar dysplasia could occur after early patellar instability [10]. In the study by Niu, the TTTG in the patellar dislocation group at the last follow-up was $3.0 \pm 0.7 \mathrm{~mm}$, while the TT-TG in the control group was $1.0 \pm 0.4 \mathrm{~mm}(P<0.05)$.

The femoral dysplasia, patellar dysplasia, and higher TT-TG were also regarded as risk factors for patellar dislocation [1-5]. So femoral dysplasia, patellar dysplasia, and high TT-TG are not only risk factors for patellar dislocation but also could be the consequences of patellar dislocation. In the present study, the aberrant femoral version was observed after patellar dislocation in the growing rabbits. Similarly, the abnormal femoral version may not only be a risk factor for patellar dislocation but also be the consequence of patellar dislocation. These findings may develop pathology and etiology of patellar instability, and emphasize the importance of the early effective treatments for patellar instability in children, considering the possibility of pathological conditions caused by femoral version deformity.

From previous studies, patellar dislocation has been successfully achieved in growing rabbits after patellar dislocation surgery $[8-10,12]$. In the present studies, patellar dislocation was observed by CT scans from each rabbit immediately after surgery and at the last followup, which showed the patellar dislocation model was obtained successfully. It is possible that alignment values have not been measured precisely because twodimensional (2D) measurements can be affected by the location of the radiation source and the limb position [4]. Recently, a three-dimensional (3D) method for measuring the alignment of the lower extremity has been widely used, which was proved to have high intraobserver and inter-observer reliability. And the method is not influenced by the femoral neck-shaft angle or postural deformity $[4,15-18]$. Considering the high accuracy of the method and the extreme flexion of the knee and hip joints in rabbits, the 3D method has been taken into account in this study and achieved high intraobserver and inter-observer reliability (Table. 3 ).

The are several limitations of the study. First, the structure of the hindlimbs of rabbits is different from human beings'. For example, adults often have femoral anteversion while mature rabbits often have femoral retroversion. On the other hand, although the rabbits had patellar dislocation surgery at one-month-old to imitate the early patellar dislocation of humans, it still could not imitate the first patellar dislocation of humans for they have abnormal knees presumably due to a genetic abnormality. So the conclusion of this study may not apply to humans. But the rabbit models have been widely used for patellar dislocation studies [8-10, 12]. The present study is the first research focusing on the influence of patellar dislocation to torsional alignment. 
It has high intra-observer and inter-observer reliability, which may enrich the etiology and pathology of patellar dislocation. Second, the knee rotation measurements were not involved in this study because of the extreme flexion in the knee joints in the rabbits. But the femoral version and tibial torsion can sufficiently reflect the torsional alignment of rabbit hindlimbs. The third limitation is the sample size of the rabbits. Although the sample size is enough according to the sample size calculation, it could be more reliable if a higher number of experimental animals were used. Also, the reason for the alteration of the femoral version at the biomechanical and molecular level should be researched in the future.

\section{Conclusions}

Based on the outcomes of this study, we conclude that early patellar dislocation can lead to the abnormal femoral version in growing rabbits. So patellar dislocation may affect lower extremity alignment.

\section{Acknowledgements}

We would like to thank Rashid Bakheit for revising manuscript.

\section{Authors' contributions}

FW designed the study. JHN, QQ, and KH performed the experimental work. $\mathrm{KH}, \mathrm{QQ}$, and $\mathrm{KP}$ evaluated the data. JHN and IS wrote the manuscript. All authors read and approved the final manuscript.

\section{Funding}

This study has been supported by the Fund for Graduates' Innovative Projects of Hebei Provincial Department of Education (No. CXZZBS2020121). The supporting of the fund: (a) the cost of CT scans (b) the cost of purchasing 30 rabbits.

\section{Availability of data and materials}

The detailed data and materials of this study are available from the corresponding author via e-mail on reasonable request..

\section{Ethics approval and consent to participate}

Institutional review board approval of the Animal Ethics Committee of the third hospital of Hebei Medical University (Number:Z2019-006-1) was obtained.

\section{Consent for publication}

Not applicable.

\section{Competing interests}

The authors declare that they have no competing interests.

\section{Author details}

'Department of joint surgery, Hebei Medical University Third Affiliated Hospital, Shijiazhaung City, Hebei Province, China. ${ }^{2}$ Department of Cardiology, Hebei Medical University Third Affiliated Hospital, Shijiazhaung City, Hebei Province, China.

Received: 22 July 2020 Accepted: 14 January 2021

Published online: 29 January 2021

\section{References}

1. Robert NS, Jared CB, Thai QT. The Prevalence and Combined Prevalences of Anatomic Factors Associated With Recurrent Patellar Dislocation. Am J Sports Med. 2015;43:921-7.

2. Dejour H, Walch G, Nove-Josserand L, Guier CH. Factors of patellar instability: an anatomic radiographic study. Knee Surg Sports Traumatol Arthrosc. 1994;2:19-26.
3. Erkocak OF, Altan E, Altintas M, Turkmen F, Aydin BK, Bayar A. Lower extremity rotational deformities and patellofemoral alignment parameters in patients with anterior knee pain. Knee Surg Sports Traumatol Arthrosc. 2016;24:3011-20.

4. Takagi S, Sato T, Watanabe S. Alignment in the transverse plane, but not sagittal or coronal plane, affects the risk of recurrent patella dislocation. Knee Surg Sports Traumatol Arthrosc. 2018;26:2891-8.

5. Diederichs G, Köhlitz T, Kornaropoulos E, Heller MO, Vollnberg B, Scheffler S. Magnetic resonance imaging analysis of rotational alignment in patients with patellar dislocations. Am J Sports Med. 2013;41:51-7.

6. Panni S, Cerciello S, Maffulli N, Di M, Servien E, Neyret P. Patellar shape can be a predisposing factor in patellar instability. Knee Surg Sports Traumatol Arthrosc. 2011;9(4):663.

7. Kaiser P, Schmoelz W, Schöttle PB, Heinrichs C, Zwierzina M, Attal R. Isolated medial patellofemoral ligament reconstruction for patella instability is insufficient for higher degrees of internal femoral torsion. Knee Surg Sports Traumatolo Arthrosc. 2019;27:758-65.

8. Li W, Wang Q, Wang F, Zhang Y, Ma L, Dong J. Femoral trochlear dysplasia after patellar dislocation in rabbits. Knee. 2013;20:485-9.

9. Wang $\mathrm{S}$, Ji G, Yang X, Wang X, Wang R, Li M. Femoral trochlear groove development after patellar subluxation and early reduction in growing rabbits. Knee Surg Sports Traumatol Arthrosc. 2016;24:247-53.

10. Jinghui N, Qi Q, Yingzhen N, Conglei D, Zhenyue D, Peng C, Fei W. Patella morphological alteration after patella instability in growing rabbits. J Orthop Surg Res. 2017;12:106.

11. Kaymaz B, Atay OA, Ergen FB, Mermerkaya MU, Olgun ZD, Atesok K. Development of the femoral trochlear groove in rabbits with patellar malposition. Knee Surg Sports Traumatol Arthrosc. 2013;21:1841-8.

12. Yingzhen N, Pengkai C, Chang L, Jinghui N, Xu Y, Fei W. Early patellar dislocation can lead to tibial tubercle lateralization in rabbits. Knee Surg Sports Traumatol Arthrosc. 2018;26:2602-6.

13. Hang F, Lisi H, Ian W, et al. Early Changes of Articular Cartilage and Subchondral Bone in The DMM Mouse Model of Osteoarthritis. Scientific reports. 2018; 8(1): 2855.

14. Gilsanz V, Roe TF, Gibbens DT, et al. Effect of sex steroids on peak bone density of growing rabbits. Am J Physiol. 1988;255:416-421.

15. JingYu J, LianYong L, LiJun Z, Qun Z, XiJuan L. Three dimensional-CT evaluation of femoral neck anteversion, acetabular anteversion and combined anteversion in unilateral DDH in an early walking age group. Inter Orthop. 2012;36:119-24

16. Riccio Al, Carney CD, Hammel LC, Stanley M, Cassidy J, Davids JR. Threedimensional computed tomography for determination of femoral anteversion in a cerebral palsy model. J Pediatr Orthop. 2015;35:167-71.

17. Ki Hyuk S, Kibeom Y, Chin Youb C, Muhammad IK. Development and Validation of a Mobile Application for Measuring Femoral Anteversion in Patients With Cerebral Palsy. J. Pediatr. Orthop. 2019. (Online ahead of print)

18. Soodmand E, Zheng G, Steens W, Bader R, Nolte L, Kluess D. Surgically Relevant Morphological Parameters of Proximal Human Femur: A Statistical Analysis Based on 3D Reconstruction of CT Data. Orthop Surg. 2019;11:135-42.

19. Liodakis E, Doxastaki I, Chu K. Reliability of the assessment of lower limb torsion using computed tomography: analysis of five different techniques. Skeletal Radiol. 2012;41:305-11.

20. Landis $\mathrm{R}$, Koch $\mathrm{G}$. The measurement of observer agreement for categorical data. Biometrics. 1977;33(1):159-74.

21. John AW. Femoral Anteversion In The Rabbit. J Bone Joint Surg. 1962;44: 386-97.

22. ChiaLing C, MengYuan T, WeiNing C, KuenHuang C. Aberrant Femoral Torsion Presenting with Frog-leg Squatting Mimicking Gluteal Muscle Contracture. Clin Orthop Relat Res. 2012:470:1165-70.

23. Fabry G, Cheng LX, Molenaers G. Normal and abnormal torsional development in children. Clin Orthop Relat Res. 1994;302:22-6.

24. Folinais D, Thelen P, Delin C, Radier C, Catonne Y, Lazennec JY. Measuring femoral and rotational alignment: EOS system versus computed tomography. Orthop Traumatol Surg Res. 2013;99:509-16.

25. Maximilian JH, Andreas P, Anne S, Daniel K, Jakob N, Johannes MR, Wolfgang L, Lars GG. Determination of Femoral Neck Angle and Torsion Angle Utilizing a Novel Three-Dimensional Modeling and Analytical Technology Based on CT Datasets. PLoS ONE. 2016;11:1-10.

26. Brouwer KJ, Molenaar JC, van Linge B. Rotational deformities after femoral shaft fractures in childhood. A retrospective study 27-32 years after the accident. Acta Orthop Scand. 1981;52:81-9. 
27. Sankar WN, Neubuerger CO, Moseley CF. Femoral anteversion in developmental dysplasia of the hip. J Pediatr Orthop. 2009:29:885-8.

28. Wines AP, MCNicol D. Computed tomography measurement of the accuracy of component version in total hip arthroplasty. J Arthrop. 2006;21:696-701.

29. Kim HT, Wenger DR. "Functional retroversion" of the femoral head in LeggCalve-Perthes disease and epiphyseal dysplasia: analysis of head-neck deformity and its effect on limb position using three-dimensional computed tomography. J Pediatr Orthop. 1997;17:240-6.

30. Yakup A, Atakan E, Muhammed BK, Osman NO, Evren A, Avni IB. Increased femoral anteversion related to infratrochanteric femoral torsion is associated with ACL rupture. Knee Surg Sports Traumatolo Arthrosc. 2020;28(8):2567-71.

31. Kaiser P, Schmoelz W, Schoettle P, Zwierzina M, Heinrichs C, Attal R. Increased internal femoral torsion can be regarded as a risk factor for patellar instability-a biomechanical study. Clin Biomech. 2017;47:103-9.

32. ZhiJun Z, Hui Z, GuanYang S, Tong Z, QianKun N, Hua F. Increased femoral anteversion is associated with inferior clinical outcomes after MPFL reconstruction and combined tibial tubercle osteotomy for the treatment of recurrent patellar instability. Knee Surg Sports Traumatol Arthrosc. 2019; 28(7):2261-9.

33. Nelitz M, Williams RS, Lippacher S, Reichel H, Dornacher D. Analysis of failure and clinical outcome after unsuccessful medial patellofemoral ligament reconstruction in young patients. Int Orthop. 2014;38:2265-72.

34. Henkel L, Willert D. A classifification and a pattern of malformation in a group of congenital defects of the limbs. J Bone Joint Surg. 1969;51:399-414.

\section{Publisher's Note}

Springer Nature remains neutral with regard to jurisdictional claims in published maps and institutional affiliations.

Ready to submit your research? Choose BMC and benefit from:

- fast, convenient online submission

- thorough peer review by experienced researchers in your field

- rapid publication on acceptance

- support for research data, including large and complex data types

- gold Open Access which fosters wider collaboration and increased citations

- maximum visibility for your research: over $100 \mathrm{M}$ website views per year

At $\mathrm{BMC}$, research is always in progress.

Learn more biomedcentral.com/submissions 